\title{
Structure-property relationships in titanium-based metal-organic frameworks for the photocatalytic reduction of carbon dioxide
}

\author{
Fernando J. Uribe-Romo \\ Assistant Professor \\ University of Central Florida \\ Orlando, FL
}

Titanium based MOFs are attractive materials for photocatalytic reduction of carbon dioxide because of the combination of high photoredox activity, high porosity, and high chemical stability. Despite the extensive amounts of research in the field of MOFs, there is a lack of understanding the principles required to establish structureproperty relationships in framework materials. This talk will describe the study of titanium MOFs based on MIL-125, in which the organic monomer has been functionalized with varied $N$-alkyl groups that systematically induce changes in photophysicial and photochemical properties due to inductive effects as well as chemical stability towards water because of the hydrophobicity of the functional group. In our study we found that MIL-125 MOFs built with secondary $N$-alkyl-2-amino-terephthalate (isopropyl, cyclopentyl, cyclohexyl) exhibit the smallest optical bandgaps (3.30 eV), with long excited-state lifetime, and apparent quantum yields up to $1.8 \%$ towards photoreduction of $\mathrm{CO}_{2}$ under blue LED light. 